\title{
Continuous Renal Replacement Therapy with oXiris Filter May Not Be an Effective Resolution to Alleviate Cytokine Release Syndrome in non-AKI Patients with Severe and Critical COVID-19
}

\author{
kai kang \\ First Affiliated Hospital of Harbin Medical University \\ yunpeng luo \\ First Affiliated Hospital of Harbin Medical University \\ yang gao \\ First Affiliated Hospital of Harbin Medical University \\ jiannan zhang \\ First Affiliated Hospital of Harbin Medical University \\ changsong wang \\ Harbin Medical University Cancer Hospital \\ dongsheng fei \\ First Affiliated Hospital of Harbin Medical University \\ wei yang \\ First Affiliated Hospital of Harbin Medical University \\ xianglin meng \\ First Affiliated Hospital of Harbin Medical University \\ ming ye \\ Second Affiliated Hospital of Harbin Medical University \\ yan gao \\ Fourth Affiliated Hospital of Harbin Medical University \\ haitao liu \\ Harbin Medical University Cancer Hospital \\ xue du \\ First Affiliated Hospital of Harbin Medical University \\ yuanyuan ji \\ First Affiliated Hospital of Harbin Medical University \\ jieling wei \\ First Affiliated Hospital of Harbin Medical University \\ wanqiu xie \\ First Affiliated Hospital of Harbin Medical University \\ jun wang \\ First Affiliated Hospital of Harbin Medical University \\ Mingyan Zhao \\ First Affiliated Hospital of Harbin Medical University \\ Kaijiang Yu ( $\nabla$ yukaijiang001@sina.com ) \\ First Affiliated Hospital of Harbin Medical University
}

\section{Research}

Keywords: continuous renal replacement therapy, oXiris filter, cytokine release syndrome, cytokine storm, hyper inflammation, non-acute kidney injury, severe and critical COVID-19, SARS-CoV-2 infection, IL-6

Posted Date: November 18th, 2021

DOI: https://doi.org/10.21203/rs.3.rs-1064997/v1

License: () (7) This work is licensed under a Creative Commons Attribution 4.0 International License. Read Full License

Version of Record: A version of this preprint was published at Frontiers in Pharmacology on February 4th, 2022. See the published version at https://doi.org/10.3389/fphar.2022.817793. 


\section{Abstract}

\section{Background}

In this study, we aimed to determine whether continuous renal replacement therapy (CRRT) with oXiris filter may alleviate cytokine release syndrome (CRS) in non-AKI patients with severe and critical coronavirus disease 2019 (COVID-19).

\section{Methods}

Non-AKI patients with severe and critical COVID-19 treated between February 14 and March 26, 2020 were included and randomly divided into intervention group and control group according to the random number table. Patients in the intervention group received CRRT with oXiris filter plus conventional treatment, while those in the control group only received conventional treatment. Demographic data were collected and collated at admission. During ICU hospitalization, the serum levels of cytokine and inflammatory chemokines, including IL-2, IL-4, IL-6, IL-10, TNF-a, and IFN-y, were measured daily to reflect the degree of CRS induced by SARS-CoV-2 infection. Clinical data, including white blood cell count (WBC), neutrophil proportion (NEUT\%), lymphocyte count (LYMPH), lymphocyte percentage (LYM\%), platelet (PLT), C-reaction protein (CRP), high sensitivity C-reactive protein (hs-CRP), alanine aminotransferase (ALT), aspartate aminotransferase (AST), total bilirubin (TB), albumin (ALB), serum creatinine (SCr), D-Dimer, fibrinogen (FIB), IL-2, IL-4, IL-6, IL-10, TNF-a, IFN-y, number of hospital days and sequential organ failure assessment (SOFA) score were obtained and collated from medical records during hospitalization, and then compared between the two groups.

\section{Results}

Age, and SCr significantly differed between the two groups. Besides the IL-2 level that was significantly lower on day 2 than that on day 1 in the intervention group, and the IL-6 levels that were significantly higher on day 1, and day 2 in the intervention group compared to the control group, similar to the IL-10 level on day 5 , there were no significant differences between the groups.

\section{Conclusion}

CRRT with oXiris filter may not effectively alleviate CRS in non-AKI patients with severe and critical COVID-19. Thus, its application in these patients should be considered with caution to avoid increasing the unnecessary burden on society and individuals and making the already overwhelmed medical system even more strained (IRB number: IRB-AF/SC-04).

\section{Background}

Coronavirus disease 2019 (COVID-19), which has wreaked havoc on the global public health system and economy, is essentially viral sepsis induced by severe acute respiratory syndrome coronavirus type 2 (SARS-CoV-2) infection, where excessive and uncontrolled systemic immune responses are the real culprit of disease deterioration and death of patients with COVID-19, and not fatal virus infection (13). This further elucidates the core reason for the current ineffectiveness of antiviral drugs used as a treatment against COVID-19 (39). SARS-CoV-2 infection can trigger the excessive production and release of a series of cytokines and inflammatory chemokines, especially marked by IL-6, IL-10, and TNF-a, also known as cytokine release syndrome (CRS) or cytokine storm, which have been revealed to be closely associated with organ injury, disease severity and death in patients with COVID-19 (8, 10, 16). Compared with serum cytokine concentrations in sputum and bronchoalveolar lavage fluid (BALF) are more representative (38). Thus, drawing lessons from the history of researches on sepsis, interventions targeting single cytokines may not be effective. However, short-term corticosteroid treatment based on the treatment concept of alleviating excessive and uncontrolled systemic immune responses is still full of controversy in clinical practice due to significant adverse effects in the middle and late stages of SARS-CoV-2 infection $(19,36)$. Consequently, it remains unclear how effective the other methods are based on controlling hyper inflammation of SARS-CoV-2 infection.

Continuous renal replacement therapy (CRRT) involves a series of treatment modalities in clinic, during which the patient's blood is drawn out of the body with an external device, and the internal environment is maintained stable based on the working principles of diffusion, convection, and membrane adsorption. The therapeutic purpose of CRRT has evolved from the single replacement of kidney function to support of multiple organ systems via the removal of cytokines and inflammatory mediators. Accordingly, it can be considered for use in acute respiratory distress syndrome (ARDS) induced by current SARS-CoV-2 infection, especially for severe and critical COVID-19 patients with CRS, regardless of the presence of acute kidney injury (AKI) complications (12, 41, 45). Continuous venovenous hemofiltration (CVVH) has occupied the mainstream of CRRT in intensive care units (ICU) due to its superior effectiveness and safety (33). In addition, regional citrate anti-coagulation is currently the main anti-coagulation method for critically ill patients requiring CRRT in clinical practice (18).

oXiris filter is a highly biocompatible modified hemodiafilter with a special heparin-coated design, which can be combined with CRRT in clinical practice. CRRT with oXiris filter can further enhance the clearance of cytokines and inflammatory chemokines, improve clinical symptoms and laboratory indicators, reduce disease severity, and prolong the survival time in critically ill patients with COVID-19 $(4,28,30)$. Still, the relevant studies mainly focused on acute or chronic renal failure in patients with COVID-19, which is one of the potential indications for CRRT initiation. Thus far, there are no sufficient clinical data to recommend the application of CRRT with oXiris filter to alleviate CRS in non-AKI patients with severe and critical COVID-19. In our study, we tried to explore the role of CRRT with oXiris filter on CRS in non-AKI patients with severe and critical COVID-19 by comparing serum cytokine and inflammatory chemokines levels during ICU hospitalization. Our findings provide a solid theoretical basis that can guide its clinical application.

\section{Methods Study design}


Non-AKI patients with severe and critical COVID-19 treated at COVID-19 treatment center of Heilongjiang province in the First Affiliated Hospital of Harbin Medical University between February 14 and March 26, 2020, were included in this prospective randomized controlled study. These patients were randomly divided into intervention group and control group according to the random number table. Patients in the intervention group received CRRT with oXiris filter plus conventional treatment, while those in the control group only received conventional treatment based on the Diagnosis and Treatment of New Coronavirus Pneumonia (the fifth edition). These patients were dealt with by the same group of experienced intensivists in the ICU.

Demographic data were collected and collated at admission. Clinical data, including white blood cell count (WBC), neutrophil proportion (NEUT\%), lymphocyte count (LYMPH), lymphocyte percentage (LYM\%), platelet (PLT), C-reaction protein (CRP), high sensitivity C-reactive protein (hs-CRP), alanine aminotransferase (ALT), aspartate aminotransferase (AST), total bilirubin (TB), albumin (ALB), serum creatinine (SCr), D-Dimer, fibrinogen (FIB), IL-2, IL-4, IL-6, IL-10, TNF-a, IFN- $\gamma$, and a number of hospital days were obtained and collated from medical records during hospitalization, and then compared between the two groups. Sequential organ failure assessment (SOFA) scores were calculated during the first $24 \mathrm{~h}$ clinical data after ICU admission.

The study was approved by the Ethics Committee of the First Affiliated Hospital of Harbin Medical University (IRB number: IRB-AF/SC-04).

\section{Study Population}

In this study, the inclusion criteria were following: (1) admitted to ICU; (2) age $\geq 18$ years old; (3) confirmed severe and critical patients with COVID-19; (4) nonAKl; (5) written informed consent obtained from patients or guardians; whereas COVID-19 patients who met the following criteria were excluded: (1) uncontrolled malignant tumors with multiple metastases; (2) leukemia; (3) acquired immunodeficiency syndrome (AIDS); (4) obstructive pneumonia caused by pulmonary tumors, severe pulmonary interstitial fibrosis, pulmonary alveolar proteinosis, and allergic alveolitis; (5) chronic organ failure; (6) immunotherapy or organ transplant within 6 months; (7) autoimmune disorder; (8) need extracorporeal membrane oxygenation (ECMO) or extracorporeal carbon dioxide removal (ECCO2R) at ICU admission; (9) patients expected to die within $72 \mathrm{~h}$; (10) pregnant or breastfeeding women; (11) incomplete medical records; (12) any potential conditions endangering the patient's safety. The full panel of experts was responsible for identifying potential conditions that could endanger the safety of enrolled patients.

\section{Diagnosis Of Severe And Critical Covid-19}

All enrolled patients were confirmed by detection of SARS-CoV-2 nucleic acid on oropharyngeal swabs, nasopharyngeal swabs, or lower respiratory tract specimens, and then classified into severe or critical cases according to the Diagnosis and Treatment of New Coronavirus Pneumonia (the fifth edition). During ICU hospitalization, the serum concentration of cytokine and inflammatory chemokines, including IL-2, IL-4, IL-6, IL-10, TNF-a, and IFN- - , were measured on a daily basis to reflect the degree of CRS induced by SARS-CoV-2 infection.

\section{Continuous Renal Replacement Therapy With Oxiris Filter}

In this study, a temporary double-lumen central venous catheter (11.5 F) was used to establish vascular access under ultrasound guidance. Patients in the intervention group were managed with $72 \mathrm{~h} \mathrm{CVVH} \mathrm{combined} \mathrm{oXiris} \mathrm{filter} \mathrm{(Baxter} \mathrm{International,} \mathrm{Deerfield,} \mathrm{IL,} \mathrm{USA),} \mathrm{regional} \mathrm{citrate} \mathrm{anti-coagulation,} \mathrm{and} \mathrm{a} \mathrm{pre-}$ and post-dilution ratio of 1:1 on a Prismaflex system (Baxter International, Deerfield, IL, USA). Blood flow rates, dehydration volume, and amount of substitute fluid were individually adjusted according to the different conditions and treatment needs of each patient. Considering the saturation of membrane adsorption, Oxiris filter was changed every 12 hours.

\section{Data Collection}

Demographic data, including age, gender, comorbidities, and clinical data, including the severity of COVID-19, SOFA score, WBC, NEUT\%, LYMPH, LYM\%, PLT, CRP, hs-CRP, ALT, AST, TB, ALB, SCr, D-Dimer, FIB, IL-2, IL-4, IL-6, IL-10, TNF-a, IFN- $y$, and a number of hospital days were obtained and collated from medical records during hospitalization by dedicated personnel in our research team. None of the other members of our research team was privy to enrolled patient's personal information beyond what was required for this study.

\section{Statistical Analyses}

SPSS 24.0 (SPSS, Inc., Chicago, IL) was used for statistical analyses. Continuous data conforming to normal distribution were described as mean \pm standard deviation (SD), while continuous data with non-normal distribution were expressed as median (P25, P75). The measurement data were expressed by frequency. Independent-samples t-test was used to perform inter-group comparison for continuous data with normal distribution, while Mann-Whitney $U$ test was employed for inter-group comparison of abnormally distributed continuous data. The Fisher's exact test was used for comparing measurement data between the two groups. P-values $<0.05$ were considered to indicate statistical significance.

\section{Results}

\section{Comparison of demographic and clinical baseline data between the two groups}


As shown in Table 1, age and SCr were significantly different in the two groups ( $p=0.026,=0.049$, respectively), despite the randomization process, while no significant difference was observed in the remaining demographic and clinical baseline data.

Table 1

Comparison of demographic and clinical baseline data between two groups

\begin{tabular}{|c|c|c|c|c|}
\hline & Intervention group & Control group & $t / z / x^{2}$ & $P$ \\
\hline Age & $67.63 \pm 9.87$ & $56.22 \pm 9.16$ & 2.470 & 0.026 \\
\hline Gender(male/female) & $7 / 1$ & $6 / 3$ & - & 0.576 \\
\hline Hypertension(yes/no) & $2 / 6$ & $4 / 5$ & - & 0.620 \\
\hline Diabetes(yes/no) & $0 / 8$ & $2 / 7$ & - & 0.471 \\
\hline Severity of COVID-19(severe/critical) & $7 / 1$ & $8 / 1$ & - & 1.000 \\
\hline SOFA score & $4(3,4)$ & $4(2.5,4.5)$ & -0.314 & 0.754 \\
\hline WBC & $6.48 \pm 2.22$ & $7.69 \pm 2.32$ & -1.099 & 0.289 \\
\hline NEUT\% & $80.98 \pm 9.71$ & $85.38 \pm 5.18$ & -1.146 & 0.278 \\
\hline LYMPH & $10.68 \pm 7.46$ & $8.01 \pm 3.84$ & 0.909 & 0.384 \\
\hline LYM\% & $0.62 \pm 0.34$ & $0.55 \pm 0.16$ & 0.473 & 0.647 \\
\hline PLT & $244.50 \pm 150.99$ & $247.89 \pm 61.98$ & -0.062 & 0.951 \\
\hline CRP & $46.58 \pm 25.65$ & $58.78 \pm 58.26$ & -0.546 & 0.593 \\
\hline hs-CRP(>10mg/L/other) & $7 / 1$ & $8 / 1$ & - & 1.000 \\
\hline ALT & $26.41(23.83,77.05)$ & $36.35(18.65,58.90)$ & -0.096 & 0.923 \\
\hline AST & $40.59 \pm 24.98$ & $37.39 \pm 27.02$ & 0.253 & 0.804 \\
\hline TB & $18.37 \pm 12.27$ & $30.13 \pm 18.83$ & -1.503 & 0.154 \\
\hline ALB & $27.67 \pm 5.39$ & $28.28 \pm 2.27$ & -0.301 & 0.770 \\
\hline $\mathrm{SCr}$ & $71.00 \pm 17.97$ & $54.73 \pm 13.30$ & 2.138 & 0.049 \\
\hline D-Dimer & $1.75(1.38,2.63)$ & $1.93(1.18,25.40)$ & -0.481 & 0.630 \\
\hline FIB & $5.84 \pm 1.30$ & $3.96 \pm 2.22$ & 2.100 & 0.053 \\
\hline IL-2 level at admission & $1.10(0.91,1.15)$ & $1.19(1.10,1.23)$ & -1.263 & 0.207 \\
\hline IL-4 level at admission & $0.59 \pm 0.32$ & $1.01 \pm 0.76$ & -1.537 & 0.153 \\
\hline IL-6 level at admission & $13.88(2.67,48.04)$ & $10.46(3.68,25.26)$ & -0.577 & 0.564 \\
\hline IL-10 level at admission & $6.71 \pm 3.97$ & $7.00 \pm 3.40$ & -0.162 & 0.873 \\
\hline TNF-a level at admission & $0.16(0.01,0.49)$ & $0.05(0,0.56)$ & -0.297 & 0.767 \\
\hline IFN-y level at admission & $0.99 \pm 0.11$ & $1.19 \pm 0.81$ & -0.734 & 0.483 \\
\hline Hospital day & $28.63 \pm 6.86$ & $28.22 \pm 6.38$ & 0.125 & 0.902 \\
\hline
\end{tabular}

Comparison of IL-2 levels between the two groups from day 1 to day 6

As shown in Table 2 and Figure 1, no difference in IL-2 levels was observed, except that the IL-2 level on day 2 was significantly lower $(P<0.05)$ than that on day 1 in the intervention group.

Table 2

Comparison of IL-2 levels between the two groups from day 1 to day 6

\begin{tabular}{|c|c|c|c|c|c|c|c|c|c|c|}
\hline Group & Day 1 & Day 2 & Day 3 & Day 4 & Day 5 & Day 6 & $\mathrm{X}^{2}{ }_{\text {Mauchy }} / \mathrm{P}$ & $F_{(G-G)} / P$ & $F_{\text {group }} / P$ & $F_{\text {group*time }} / I$ \\
\hline $\begin{array}{l}\text { Control } \\
\text { group }\end{array}$ & $1.5 \pm 0.62$ & $0.98 \pm 0.70$ & $0.97 \pm 0.50$ & $1.20 \pm 0.73$ & $1.17 \pm 0.85$ & $1.48 \pm 0.84$ & $27.95 / 0.016$ & $3.979 / 0.012$ & $1.069 / 0.318$ & $0.854 / 0.74 !$ \\
\hline $\begin{array}{l}\text { Intervention } \\
\text { group }\end{array}$ & $1.68 \pm 0.63$ & $0.86 a \pm 0.47$ & $0.52 \pm 0.37$ & $0.68 \pm 0.76$ & $1.49 \pm 0.89$ & $1.43 \pm 0.84$ & & & & \\
\hline
\end{tabular}

a Represent significant difference compared with day 1. 
As shown in Table 3 and Figure 2, no significant difference in IL-4 levels was observed between the two groups from day 1 to day 6.

Table 3

Comparison of IL-4 levels between the two groups from day 1 to day 6

\begin{tabular}{|c|c|c|c|c|c|c|c|c|c|c|}
\hline & Day 1 & Day 2 & Day 3 & Day 4 & Day 5 & Day 6 & $\mathrm{X}^{2}{ }_{\text {Mauchy }} / \mathrm{P}$ & $F_{(G-G)} / P$ & $F_{\text {group }} / P$ & 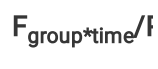 \\
\hline $\begin{array}{l}\text { Control } \\
\text { group }\end{array}$ & $1.36 \pm 0.44$ & $0.55 \pm 0.49$ & $0.94 \pm 1.05$ & $1.41 \pm 0.89$ & $1.77 \pm 1.19$ & $1.35 \pm 0.93$ & $32.784 / 0.004$ & $1.854 / 0.152$ & $0.032 / 0.861$ & $0.385 / 0.76:$ \\
\hline $\begin{array}{l}\text { Intervention } \\
\text { group }\end{array}$ & $1.08 \pm 0.70$ & $0.78 \pm 0.68$ & $1.07 \pm 1.13$ & $1.17 \pm 1.42$ & $1.38 \pm 1.41$ & $1.70 \pm 1.21$ & & & & \\
\hline
\end{tabular}

\section{Comparison of IL- 6 levels between the two groups from day 1 to day 6}

As shown in Table 4 and Figure 3, no significant difference was found except that the IL- 6 levels on day 1 and 2 in the intervention group were significantly higher $(P<0.05)$ than those in the control group.

Table 4

Comparison of IL- 6 levels between the two groups from day 1 to day 6

\begin{tabular}{|c|c|c|c|c|c|c|c|c|c|c|}
\hline & Day 1 & Day 2 & Day 3 & Day 4 & Day 5 & Day 6 & $\mathrm{X}^{2}{ }_{\text {Mauchy }} / \mathrm{P}$ & F/P & $F_{\text {group }} / P$ & $F_{\text {group*tim }}$ \\
\hline $\begin{array}{l}\text { Control } \\
\text { group }\end{array}$ & $1.99 \pm 0.99$ & $1.22 \pm 2.37$ & $2.31 \pm 0.99$ & $2.82 \pm 1.46$ & $2.04 \pm 0.91$ & $2.07 \pm 1.22$ & $20.540 / 0.119$ & $1.906 / 0.103$ & $5.972 / 0.027$ & $1.710 / 0$ \\
\hline $\begin{array}{l}\text { Intervention } \\
\text { group }\end{array}$ & $3.41 \mathrm{a} \pm 0.78$ & $3.25 a \pm 0.99$ & $2.93 \pm 1.34$ & $3.42 \pm 0.62$ & $3.24 \pm 1.15$ & $3.20 \pm 1.08$ & & & & \\
\hline
\end{tabular}

${ }^{a}$ Represent significant difference compared with the control group

\section{Comparison of IL-10 levels between the two groups from day 1 to day 6}

As shown in Table 5 and Figure 4, no significant difference was observed except that the IL-10 levels on day 5 in the intervention group were significantly higher $(P<0.05)$ than the control group.

Table 5

Comparison of IL-10 levels between the two groups from day 1 to day 6

\begin{tabular}{|c|c|c|c|c|c|c|c|c|c|c|}
\hline & Day 1 & Day 2 & Day 3 & Day 4 & Day 5 & Day 6 & $\mathrm{X}^{2}{ }_{\text {Mauchy }} / \mathrm{P}$ & $F_{(G-G)} / P$ & $F_{\text {group }} / P$ & $F_{\text {group* }}$ \\
\hline $\begin{array}{l}\text { Control } \\
\text { group }\end{array}$ & $7.45 \pm 4.75$ & $5.39 \pm 2.19$ & $7.46 \pm 4.75$ & $6.59 \pm 2.53$ & $5.20 \pm 1.74$ & $5.57 \pm 2.40$ & $30.709 / 0.007$ & $0.371 / 0.521$ & $6.178 / 0.025$ & $0.474 /$ \\
\hline $\begin{array}{l}\text { Intervention } \\
\text { group }\end{array}$ & $9.33 \pm 5.18$ & $7.32 \pm 3.80$ & $10.67 \pm 9.19$ & $9.54 \pm 4.01$ & $9.37 a \pm 4.65$ & $11.70 \pm 10.29$ & & & & \\
\hline
\end{tabular}

${ }^{\text {a }}$ Represent significant difference compared with the control group

\section{Comparison of TNF-a levels between the two groups from day 1 to day 6}

As shown in Table 6 and Figure 5, no significant differences were found in TNF-a levels when comparing the two groups from day 1 to day 6.

Table 6

Comparison of TNF-a levels between the two groups from day 1 to day 6

\begin{tabular}{|c|c|c|c|c|c|c|c|c|c|c|}
\hline & Day 1 & Day 2 & Day 3 & Day 4 & Day 5 & Day 6 & $\mathrm{X}^{2}$ Mauchy/P & $F_{(G-G)} / P$ & $F_{\text {group }} / P$ & $F_{\text {group*time }} / I$ \\
\hline $\begin{array}{l}\text { Control } \\
\text { group }\end{array}$ & $1.14 \pm 0.26$ & $0.73 \pm 0.36$ & $1.50 \pm 1.53$ & $1.50 \pm 1.10$ & $0.90 \pm 1.10$ & $1.02 \pm 0.62$ & $42.026 / 0.000$ & $2.096 / 0.116$ & $0.003 / 0.959$ & $1.857 / 0.31 €$ \\
\hline $\begin{array}{l}\text { Intervention } \\
\text { group }\end{array}$ & $0.93 \pm 0.24$ & $0.65 \pm 0.18$ & $0.69 \pm 0.41$ & $0.82 \pm 0.55$ & $2.36 \pm 1.59$ & $1.40 \pm 0.73$ & & & & \\
\hline
\end{tabular}

\section{Comparison of IFN-y levels between the two groups from day 1 to day 6}

As shown in Table 7 and Figure 6, no significant difference was found in IFN-ץ levels when comparing the two groups from day 1 to day 6. 
Table 7

Comparison of IFN-y levels between the two groups from day 1 to day 6

\begin{tabular}{|c|c|c|c|c|c|c|c|c|c|c|}
\hline & Day 1 & Day 2 & Day 3 & Day 4 & Day 5 & Day 6 & $\mathrm{X}^{2}{ }_{\text {Mauchy }} / \mathrm{P}$ & $F_{(G-G)} / P$ & $F_{\text {group }} / P$ & $F_{\text {group }}{ }^{\star t i m e}$ \\
\hline $\begin{array}{l}\text { Control } \\
\text { group }\end{array}$ & $1.39 \pm 0.32$ & $1.14 \pm 0.34$ & $1.26 \pm 0.57$ & $1.52 \pm 0.68$ & $1.44 \pm 0.61$ & $1.44 \pm 0.88$ & $110.437 / 0.000$ & $2.043 / 0.162$ & $1.613 / 0.223$ & $1.227 / 0.31$ \\
\hline $\begin{array}{l}\text { Intervention } \\
\text { group }\end{array}$ & $1.34 \pm 0.37$ & $1.07 \pm 0.38$ & $1.22 \pm 0.47$ & $2.04 \pm 1.51$ & $2.27 \pm 1.95$ & $3.10 \pm 4.00$ & & & & \\
\hline
\end{tabular}

\section{Discussion}

Nearly two years have passed since the outbreak of COVID-19 in Wuhan; however, the disease is still rapidly advancing around the world, with the number of SARS-CoV-2 infections and death reaching new records every day. As a novel, highly pathogenic human coronavirus (hCoV), SARS-CoV-2 may continue to pose a persistent and unprecedented threat to global public health security for a considerable time to come. Breakthrough infections caused by adaptive mutations in the SARS-COV-2 genome prevent universal vaccination from becoming the effective coping strategy against COVID-19 (14). In the absence of available targeted interventions, there is an urgent need to explore effective treatment approaches based on an ongoing understanding of the pathogenesis of SARS-CoV-2 viral infection and disease deterioration to avert irreversible multi-organ failure induced by SARS-CoV-2 infection in clinical practice, especially for severe and critical COVID-19 patients with high mortality. Among them, CRRT with oXiris filter is a highly expected and promising clinical approach (6).

Nearly $20 \%$ of patients with COVID-19 tend to develop into severe and critical cases, which usually involve the lungs and manifest as acute respiratory distress syndrome (ARDS) and marked dysregulated immunity (40). Although the exact pathogenesis of SARS-CoV-2 infection and disease deterioration is still poorly understood, CRS characterized by an excessive and uncontrolled systemic inflammatory response has an essential role (21). A significant increase in cytokines and inflammatory chemokines burden was observed in patients with COVID-19, in association with multiple-organ dysfunction, increased disease severity, and adverse clinical outcomes $(16,34,44)$. Data from the Chinese Center for Disease Control and Prevention indicated that the overall mortality of COVID-19 was $2.3 \%$, while the mortality of patients with critical COVID- 19 was close to $50 \%$ and even exceeded $60 \%$ in the early stages of the outbreak ( 40 , 42). Some of the survivors, who recovered from severe or critical COVID-19, were reported to suffer from severely impaired pulmonary diffusion capacities and abnormal chest imaging manifestations at 6-months follow-up after SARS-CoV-2 infection (15). It is a realistic clinical need to alleviate organ damage and reduce mortality in patients with severe and critical COVID-19. Regulating inflammatory responses to restore immunological equilibrium and maintain immune homeostasis may be an entry point strategy.

Obviously, organ support therapy alone is not enough for CRS-induced organ injury in patients with COVID-19. Using CRRT for immunomodulation has a long history in clinic for tapering cytokine storms and controlling the associated dysregulation of the immune system. This approach has also been proposed as adjuvant therapy in many diseases, including sepsis (22), septic AKI (35), septic shock (32), severe Middle East Respiratory Syndrome (MERS) (5), severe acute pancreatitis (SAP) with or without ARDS $(9,11)$, CRS induced by some immunotherapies (7), severe burns (27), etc. Yet, a non-selective way allows harmful and beneficial substances to be simultaneously removed during CRRT (33). Moreover, the potential disadvantages of CRRT, such as technical complications of establishing vascular access, anti-coagulation-related complications, hemodynamic instability, internal environment disturbance, obstacles to the spontaneous recovery of renal function, and huge cost, should not be ignored in clinical practice. As a result, although some studies have confirmed the immunomodulation effect of CRRT, this was not sufficient to influence clinical endpoints and could even prolong the need for organ support (25, 43).

oXiris filter is superficially modified from an AN-69 membrane (polyacrylonitrile) with an additional positively charged polyimide ethylene layer used to enhance the cytokines-adsorbing capacity by ionic bonding and grafted with heparin (3), which was first approved and marketed in Europe in 2009. The addition of the highly adsorptive preheparinized oXiris filter can enhance the ability of CRRT to effectively remove endotoxin, circulating cytokines, and inflammatory chemokines, thus reducing lactate concentration and vasopressors infusion rate, and improving haemodynamic status, systemic perfusion, multi-organ function, and clinical outcomes without related adverse events $(3,31,32,35,37)$. Therefore, it gained emergency approval from US Food and Drug Administration (FDA) for the clinical treatment of COVID-19 in April 2020 to counter the CRS attack triggered by SARS-CoV-2 infection. Different from shortterm corticosteroid treatment, CRRT with oXiris filter is expected to mitigate circulating cytokines and inflammatory chemokines burden and restore immune homeostasis in COVID-19 patients without a prolonged state of immunosuppression and serious secondary infection.

In our study, CRRT with oXiris filter showed no advantage in removing circulating cytokines and inflammatory chemokines in non-AKI patients with severe and critical COVID-19. The primary reason is that all the selected patients were non-AKI with normal renal clearance, which is significantly different from previous relevant studies that mainly focused on the acute or chronic renal failure patients with seriously damaged renal clearance. In such case, CRRT with or without oXiris filter becomes an important way to clear circulating and inflammatory chemokines. In addition, the levels of IL-6 in patients with severe COVID-19 was usually tens of $\mathrm{pg} / \mathrm{mL}$, which was similar to our results and far lower than those in patients with septic shock or CRS induced by Chimeric Antigen Receptor (CAR) T-cell infusion $(1,23)$. A previous study has confirmed that the immunomodulatory treatment of Afelimomab exerts a protective effect in patients with severe sepsis only when the level of IL-6 exceeds the threshold of $1000 \mathrm{pg} / \mathrm{mL}$ (24). The level of IL-6 in our study, which was far from reaching or close to this threshold, may explain these negative results. Furthermore, different inflammatory subphenotypes of COVID-19 may have a certain impact on the production and release of circulating cytokines and inflammatory chemokines (17). Lastly, changes in circulating cytokines and inflammatory chemokines levels may not be solely influenced by extracorporeal removal, but also by SARS-CoV-2 viral load, endogenous production, innate and acquired immune defense, comorbidities and many other factors $(2,20,26,29)$.

There are some limitations in the present study. First of all, as this was a small-size single-center study, the possible positive results may be hidden after increasing the number of cases, and the credibility and generalizability of our conclusion may weaken. Second, the duration varies from onset to ICU 
admission, which may influence the production and removal of circulating cytokines and inflammatory chemokines to a certain extent, although no significant difference was observed in the clinical baseline data between the two groups except Scr. Third, the monitoring duration of cytokines and inflammatory chemokines levels was limited to 6 days, and thus the medium and long term role of CRRT with oXiris filter in non-AKI patients with severe and critical COVID19 was not further explored. Finally, the types of cytokines and inflammatory chemokines detection were also limited.

\section{Conclusion}

CRRT with oXiris filter may not be an effective method for alleviating CRS in non-AKI patients with severe and critical COVID-19; thus, its application in these patients should be considered with caution to avoid increasing the unnecessary burden on society and individuals and making the already overwhelmed medical system even more overstretched. The findings of our study need to be further confirmed by well-designed large-sample studies.

\section{Declarations}

\section{Acknowledgments}

The authors are grateful to all colleagues who worked with them in the COVID-19 treatment center of Heilongjiang province, and all those who provided selfless advice and help for this article. We pay tribute to the medical staff who lost their lives in the national fight against the COVID-19 epidemic.

\section{Ethics Approval and Consent to Participate}

Written informed consent was duly obtained from all enrolled patients. The study was approved by the Ethics Committee of the First Affiliated Hospital of Harbin Medical University (IRB-AF/SC-04), and registered at clinicaltrials.gov.cn (ChiCTR2000030265).

\section{Competing interests}

The authors declare that they have no competing interests.

\section{Authors' Contributions}

K.K., Y.L., Y.G., J.Z., C.W., M.Z., and K.Y. took part in the conception, literature search, study design, statistical analysis, analysis and discussion of results, and manuscript preparation, editing, and review. D.F., W.Y., X.M., M.Y., Y.G., H.L., X.D., Y.J., J.W., W.X., and J.W. provided assistance for the conception, literature search, data acquisition and collation, statistical analysis, analysis and discussion of results, and manuscript preparation. All authors read and approved the final article.

\section{Funding}

This study was supported by the National Natural Science Foundation of China (Nos. 81770276, 81772045, 81902000) and Nn10 program of Harbin Medical University Cancer Hospital.

\section{Consent for publication}

All authors read and approved the final version for publication.

\section{Availability of data and materials}

The datasets used and/or analyzed during the current study are available from the corresponding author on reasonable request.

\section{References}

1. Aziz M, Fatima R, Assaly R. Elevated interleukin-6 and severe COVID-19: A meta-analysis. J Med Virol 2020;92:2283-2285.

2. Bermejo-Martin JF, González-Rivera M, Almansa R, et al. Viral RNA load in plasma is associated with critical illness and a dysregulated host response in COVID-19. Crit Care 2020;24(1):691.

3. Broman ME, Hansson F, Vincent JL, et al. Endotoxin and cytokine reducing properties of the oXiris membrane in patients with septic shock: A randomized crossover double-blind study. PLoS One 2019;14:e0220444.

4. Cascarano L, Cutuli SL, Pintaudi G, et al. Extracorporeal immune modulation in COVID-19 induced immune dysfunction and secondary infections: the role of oXiris $\AA$ membrane. Minerva Anestesiol 2021;87:384-385.

5. Cha RH, Joh JS, Jeong I, et al. Renal Complications and Their Prognosis in Korean Patients with Middle East Respiratory Syndrome-Coronavirus from the Central MERS-CoV Designated Hospital. J Korean Med Sci 2015;30:1807-1814.

6. Chen G, Zhou Y, Ma J, et al. Is there a role for blood purification therapies targeting cytokine storm syndrome in critically severe COVID-19 patients? Ren Fail 2020;42:483-488.

7. Constantinescu C, Pasca S, Tat T, et al. Continuous renal replacement therapy in cytokine release syndrome following immunotherapy or cellular therapies? J Immunother Cancer 2020;8:e000742.

8. Copaescu A, Smibert 0, Gibson A, et al. The role of IL-6 and other mediators in the cytokine storm associated with SARS-CoV-2 infection. J Allergy Clin Immunol 2020;146:518-534.e1. 
9. Cui HX, Xu JY, Li MQ. Efficacy of continuous renal replacement therapy in the treatment of severe acute pancreatitis associated acute respiratory distress syndrome. Eur Rev Med Pharmacol Sci 2014;18:2523-2526.

10. Del Valle DM, Kim-Schulze S, Huang HH, et al. An inflammatory cytokine signature predicts COVID-19 severity and survival. Nat Med 2020;26:1636-1643.

11. Gao N, Yan C, Zhang G. Changes of Serum Procalcitonin (PCT), C-Reactive Protein (CRP), Interleukin-17 (IL-17), Interleukin-6 (IL-6), High Mobility Group Protein-B1 (HMGB1) and D-Dimer in Patients with Severe Acute Pancreatitis Treated with Continuous Renal Replacement Therapy (CRRT) and Its Clinical Significance. Med Sci Monit 2018;24:5881-5886.

12. Gao Y, Qi ZD, Liu RJ, et al. A multi-center cross-sectional study on blood purification among adult patients in intensive care unit in China: a study protocol. Chin Med J (Engl) 2019;132:1208-1211.

13. Gao Y, Wang C, Kang K, et al. Cytokine Storm May Not Be the Chief Culprit for the Deterioration of COVID-19. Viral Immunol 2021;34:336-341.

14. Hacisuleyman E, Hale C, Saito Y, et al. Vaccine Breakthrough Infections with SARS-CoV-2 Variants. N Engl J Med 2021;384:2212-2218.

15. Huang C, Huang L, Wang Y, et al. 6-month consequences of COVID-19 in patients discharged from hospital: a cohort study. Lancet 2021;397:220-232.

16. Huang C, Wang Y, Li X, et al. Clinical features of patients infected with 2019 novel coronavirus in Wuhan, China. Lancet 2020;395:497-506.

17. Lin SH, Zhao YS, Zhou DX, et al. Coronavirus disease 2019 (COVID-19): cytokine storms, hyper-inflammatory phenotypes, and acute respiratory distress syndrome. Genes Dis 2020;7:520-527.

18. Liu C, Mao Z, Kang H, et al. Regional citrate versus heparin anticoagulation for continuous renal replacement therapy in critically ill patients: a metaanalysis with trial sequential analysis of randomized controlled trials. Crit Care 2016;20:144.

19. Liu Z, Li X, Fan G, et al. Low-to-moderate dose corticosteroids treatment in hospitalized adults with COVID-19. Clin Microbiol Infect 2021;27:112-117.

20. Lumlertgul N, Hall A, Camporota L, et al. Clearance of inflammatory cytokines in patients with septic acute kidney injury during renal replacement therapy using the EMiC2 filter (Clic-AKI study). Crit Care 2021;25:39.

21. Mehta P, McAuley DF, Brown M, et al. COVID-19: consider cytokine storm syndromes and immunosuppression. Lancet 2020;395:1033-1034.

22. Monard C, Rimmelé T, Ronco C. Extracorporeal Blood Purification Therapies for Sepsis. Blood Purif 2019;47 Suppl 3:1-14.

23. Monneret G, Benlyamani I, Gossez M, et al. COVID-19: What type of cytokine storm are we dealing with? J Med Virol 2021;93:197-198.

24. Panacek EA, Marshall JC, Albertson TE, et al. Efficacy and safety of the monoclonal anti-tumor necrosis factor antibody F(ab')2 fragment afelimomab in patients with severe sepsis and elevated interleukin-6 levels. Crit Care Med 2004;32:2173-2182.

25. Park JT, Lee H, Kee YK, et al. High-Dose Versus Conventional-Dose Continuous Venovenous Hemodiafiltration and Patient and Kidney Survival and Cytokine Removal in Sepsis-Associated Acute Kidney Injury: A Randomized Controlled Trial. Am J Kidney Dis 2016;68:599-608.

26. Pasrija R, Naime M. The deregulated immune reaction and cytokines release storm (CRS) in COVID-19 disease. Int Immunopharmacol 2021;90:107225.

27. Peng Y, Yuan Z, Li H. Removal of inflammatory cytokines and endotoxin by veno-venous continuous renal replacement therapy for burned patients with sepsis. Burns 2005;31:623-628.

28. Premužić V, Babel J, Gardijan D, et al. Extracorporeal blood purification is associated with improvement in biochemical and clinical variables in the critically-ill COVID-19 patients. Ther Apher Dial 2021. Epub ahead of print.

29. Rajpal A, Rahimi L, Ismail-Beigi F. Factors leading to high morbidity and mortality of COVID-19 in patients with type 2 diabetes. J Diabetes 2020;12:895908.

30. Rosalia RA, Ugurov P, Neziri D, et al. Extracorporeal Blood Purification in Moderate and Severe COVID-19 Patients: A Prospective Cohort Study. Blood Purif 2021:1-10. Epub ahead of print.

31. Samman KN, Baalbaki H, Bouchard J, et al. Continuous Renal Replacement Therapy with oXiris ${ }^{\circledR}$ Membrane in Severe Ebstein-Barr Virus-Mediated Hemophagocytic Lymphohistiocytosis: A Case Report. Blood Purif 2021;50:578-581.

32. Schwindenhammer V, Girardot T, Chaulier K, et al. oXiris $\AA^{2}$ Use in Septic Shock: Experience of Two French Centres. Blood Purif 2019;47 Suppl 3:1-7.

33. Shi N, Sun GD, Ji YY, et al. Effects of acute kidney injury on acute pancreatitis patients' survival rate in intensive care unit: A retrospective study. World J Gastroenterol 2021; 27: 6453-6464.

34. Sims JT, Krishnan V, Chang CY, et al. Characterization of the cytokine storm reflects hyperinflammatory endothelial dysfunction in COVID-19. J Allergy Clin Immunol 2021;147:107-111.

35. Turani F, Barchetta R, Falco M, et al. Continuous Renal Replacement Therapy with the Adsorbing Filter oXiris in Septic Patients: A Case Series. Blood Purif 2019;47 Suppl 3:1-5.

36. van Paassen J, Vos JS, Hoekstra EM, et al. Corticosteroid use in COVID-19 patients: a systematic review and meta-analysis on clinical outcomes. Crit Care 2020;24:696.

37. Villa G, Romagnoli S, De Rosa S, et al. Blood purification therapy with a hemodiafilter featuring enhanced adsorptive properties for cytokine removal in patients presenting COVID-19: a pilot study. Crit Care 2020;24:605.

38. Wang C, Kang K, Lan X, et al. Cytokine levels in sputum, not serum, may be more helpful for indicating the damage in the lung and the prognosis of severe COVID-19 - A case series. J Infect 2021;S0163-4453:00415-1.

39. WHO Solidarity Trial Consortium, Pan H, Peto R, et al. Repurposed Antiviral Drugs for Covid-19 - Interim WHO Solidarity Trial Results. N Engl J Med 2021;384:497-511.

40. Wu Z, McGoogan JM. Characteristics of and Important Lessons From the Coronavirus Disease 2019 (COVID-19) Outbreak in China: Summary of a Report of 72314 Cases From the Chinese Center for Disease Control and Prevention. JAMA 2020;323:1239-1242. 
41. Xiang H, Song B, Zhang Y, et al. The effectiveness of continuous renal replacement therapy in critical COVID-19 patients with cytokine release syndrome: a retrospective, multicenter, descriptive study from Wuhan, China. Aging (Albany NY) 2021;13(7):9243-9252.

42. Yang $X, Y u Y, X u$ J, et al. Clinical course and outcomes of critically ill patients with SARS-CoV-2 pneumonia in Wuhan, China: a single-centered, retrospective, observational study. Lancet Respir Med 2020;8(5):475-481.

43. Yin F, Zhang F, Liu S, et al. The therapeutic effect of high-volume hemofiltration on sepsis: a systematic review and meta-analysis. Ann Transl Med 2020;8:488.

44. Zeng F, Huang Y, Guo Y, et al. Association of inflammatory markers with the severity of COVID-19: A meta-analysis. Int J Infect Dis 2020;96:467-474.

45. Zhang H, Zhu G, Yan L, et al. The absorbing filter Oxiris in severe coronavirus disease 2019 patients: A case series. Artif Organs 2020;44:1296-1302.

\section{Figures}

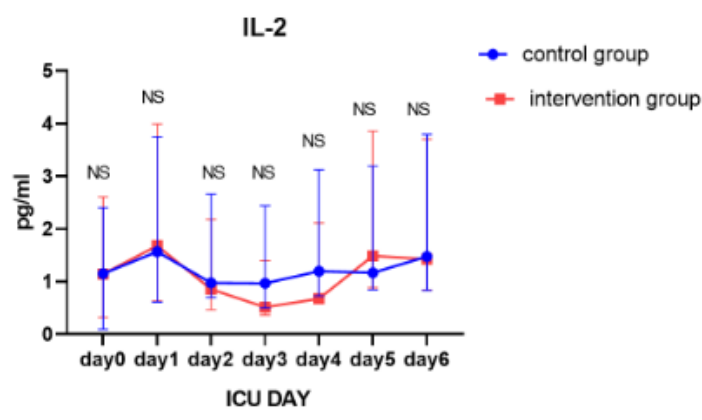

\section{Figure 1}

Longitudinal comparison of IL-2 levels between the two groups from day 1 to day 6 .

IL-4

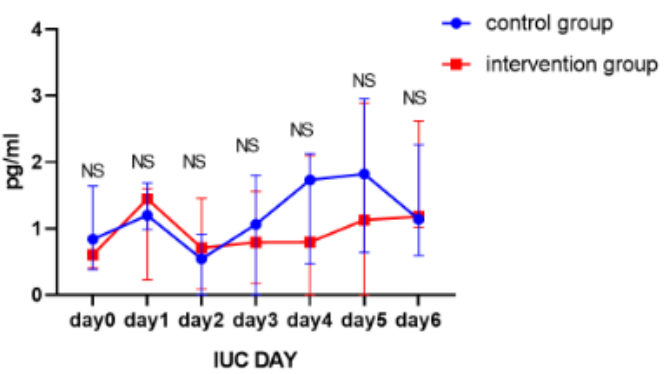

Figure 2

Longitudinal comparison of IL-4 levels between the two groups from day 1 to day 6 .

IL-6

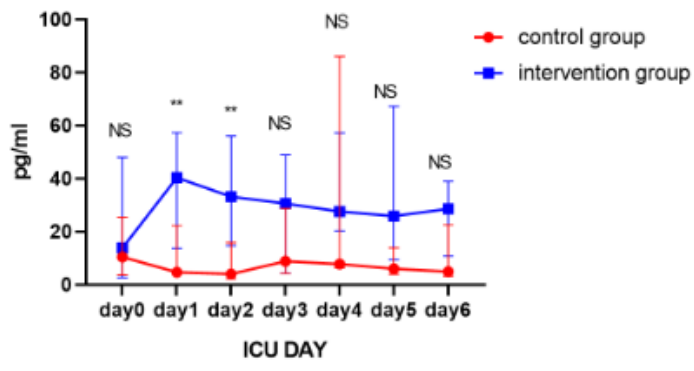

Figure 3

Longitudinal comparison of IL-6 levels between the two groups from day 1 to day 6 . 


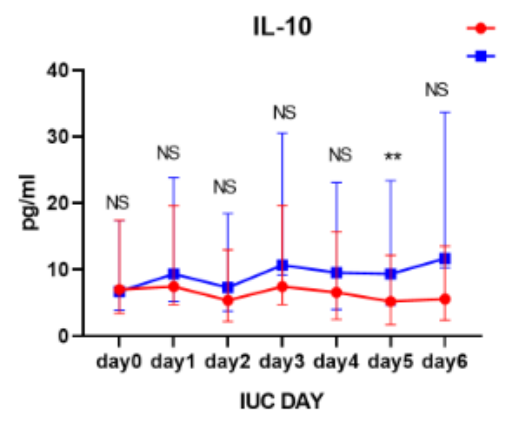

\section{Figure 4}

Longitudinal Comparison of IL-10 levels between the two groups from day 1 to day 6 .

TNF- $\alpha$

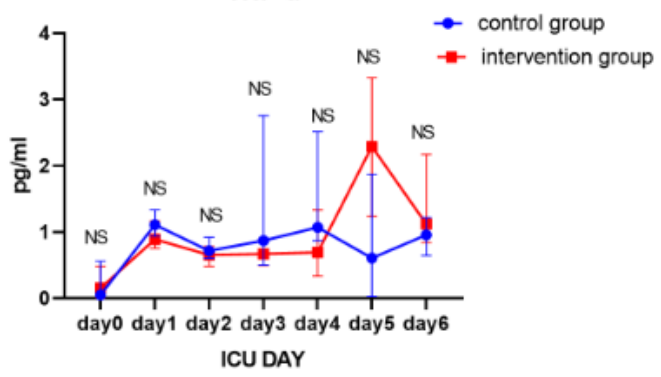

Figure 5

Longitudinal comparison of TNF-a levels between the two groups from day 1 to day 6 .

IFN-Y

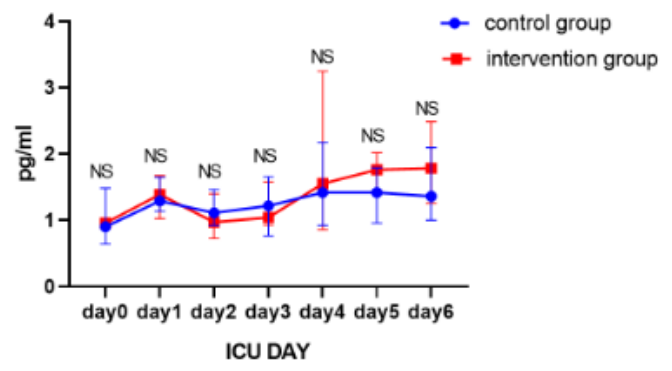

\section{Figure 6}

Longitudinal comparison of IFN-y levels between the two groups from day 1 to day 6 